\title{
Customer profile analysis of the mail- order pharmacy MedicineGo using the example of the German mail-order pharmacy MedicineGo
}

\author{
Tim Gaebert \\ Mendel University; Brno, Czech Republic
}

\begin{abstract}
Pharmacies in transition - digitalisation measures, connection to the telematics infrastructure and the oligopoly position of leading mail-order pharmacies. Not only meeting the technical implementations in the digital transformation, but also satisfying customers are important issues for public pharmacies. While retail pharmacies have access to data on their regular customers, we know little about customers of mail-order pharmacies. As the trend to order medicines online grows, mail-order medicines will take on an increasingly important role. It is expected that the planned introduction of electronic prescriptions in Germany in mid-2021 will lead to a further increase in online sales.

The research question is: What does the customer profile of a mail-order pharmacy look like? Thus, this study focuses on an economic customer profiling of a mail-order pharmacy using the example of the nationally operating mail-order pharmacy MedicineGo. Based on the assumption that younger people between 18 and 50 years of age prefer to use the internet as an ordering medium, due to a savvier use of computers, a quantitative analysis of empirical data will present essential market research data. To interpret the results of the quantitative analysis and to establish causalities, additional expert interviews with the MedicineGo administrator and other employees of the mailorder pharmacy as well as a qualitative literature analysis were conducted by means of qualitative analysis.
\end{abstract}


By means of quantitative analysis, it was empirically shown that regarding the customer profile of the mail-order pharmacy MedicineGo, $72 \%$ of customers use the direct web shop as a sales channel for their orders, the proportion of women is about ten percentage points higher than that of men, and the largest purchasing groups by number of orders are persons between 51 and 80 years of age. The quantitative analysis showed that older people (over 50 years) prefer to use the mail-order pharmacy, so another result of the study is that public pharmacies compete with mail-order pharmacies for the same customer group.

Keywords: pharmacy customers, mail order pharmacy, medicines, customer profile mail order pharmacy.

JEL code: C46, I11

\section{Introduction}

Figures from the German Pharmacists' Association (ABDA) show a continuous decline in the number of pharmacies in Germany. (ABDA) show a continuous decline in the number of pharmacies in Germany. Since the all-time high of 2008 with 21,602 pharmacies, pharmacies have been closing continuously (see Fig. 1). According to the industry communication platform apotheke adhoc, pharmacies are also closing faster and faster. In 2012, there were under 21,000 pharmacies in Germany, in 2017 under 20,000 and at the end of the first quarter of 2020 under 19,000 (apotheke adhoc, 2020, 1).

Large and internationally active mail-order pharmacies are enjoying rising sales. Shop Apotheke Europe, for example, was able to increase its turnover by 41 per cent from 126 million euros (2015) to 177 million euros (2016) within one year (Tebroke, 2017, 8). While foreign mail-order companies are experiencing a boom in sales, the European Court of Justice overturned the price maintenance for foreign mail-order pharmacies in its ruling of 19 October 2016 - a competitive disadvantage for German retail pharmacies, which are subject to price maintenance for prescription medicines and are not allowed to give bonuses on Rx preparations (AMPreisV) (ECJ, judgment of 19 October 2016, Deutsche Parkinson Vereinigung e.V., C-148/15).

Thus, public pharmacies are under increasing pressure due to ever stronger online competition, especially from mail-order giants, and a general increase in the trend to order medicines online.

So far, there is a lack of online customer profiling of mail order pharmacies so that retail pharmacies can compete with this target group and create incentives and offers. 


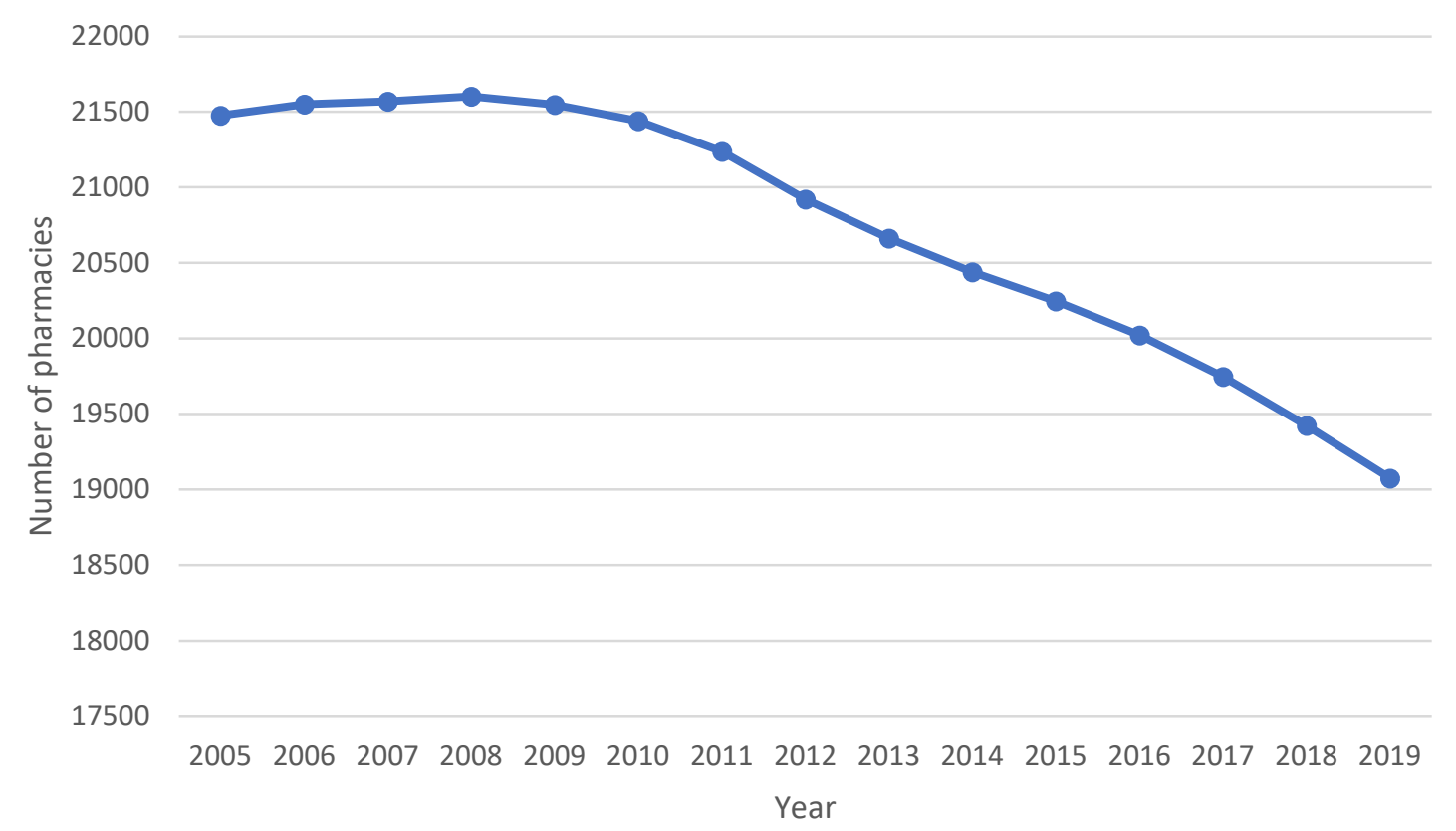

Fig. 1: Development of the number of pharmacies in Germany.

Source: ABDA, 2020, 13.

\section{Targets}

The current academic literature deals with the ECJ ruling of 19.10.2016, in which it allows foreign mail-order pharmacies to grant discounts on prescription medicines, while German (mail-order) pharmacies are subject to price maintenance and are not allowed to give bonuses. In this regard, pharmaceutical law expert Dettling has outlined models in his analysis of union and constitutional law that restore this imbalance between foreign mail-order pharmacies and national retail pharmacies (Dettling, 2018, 63). Other academic articles focus on the planned introduction of electronic prescriptions in Germany in mid-2021 (ZTG, 2020). For example, in his e-prescription study of 2019, Kaske analyses possible consequences after the e-prescription introduction and the subsequent ten years for the pharmacy landscape (Kaske, 2019). However, despite the growing trend to order medicines online, there are no studies that define the online medicine customer. Most articles are limited to sales figures from leading mail-order pharmacies that confirm the online trend.

The main objective of this paper is to create an economic customer profile of the mailorder pharmacy MedicineGo based on empirical data using quantitative methods. The profiling includes the following sub-objectives:

- Overview creation of the sales channel usage

- Overview of order shares according to age specification

- Gender-specific share and differences in ordering behaviour

The research question is: What does the customer profile look like for a mail order company for pharmaceuticals? For this purpose, the following hypothesis is made: When customers order medicines online, they are mainly between 18 and 50 years of age, based on the assumption that younger people (18 to 50 years) prefer to order online, due to a savvy computer use. 
While the quantitative method presents results on the customer profile, the expert interviews also provide causalities and background to the results from the quantitative method by means of qualitative analysis in combination with a qualitative literature analysis.

On the basis of the study, the results can show optimisations for the pharmacies in private practice, so that the market research data represent a benefit for the retail pharmacies. For example, the characterisation of online customers in the pharmaceutical segment can reveal essential economic aspects in e-commerce, especially for the pharmaceutical market, and can be used as basic data for pharmacies for optimised marketing.

\section{Methodological approach and data}

To answer the research question and the subdivided sub-objectives, an empirical quantitative and qualitative approach was taken by means of own data collection, ten expert interviews and a literature analysis.

The basic data for answering the research question was the data collection of the raw data from MedicineGo, this took place on 10.01.2017 and covers the period from 05.08.2014 to 31.12.2016. Due to new operating systems, data before 05.08.2014 is not tangible. For this purpose, the empirical data was evaluated quantitatively; the total number of evaluated customer orders comprises approx. 22,000 billing data.

With the empirical study, based on routine data (billing data) of the German mail order pharmacy MedicineGo, a flexible database was created. For operation and data backup, the billing data of online pharmacy customers are stored on the online server Mauve. The data from Mauve was exported to an external non-flexible database (Mauve System3). For this study, data from Mauve System3 was exported to cvs (commaseparated values) text file and then imported into an SQLite database created for this purpose. In this way, the cvs files could be represented by means of transfer and postprocessing, for example in Microsoft Excel, and recorded by measurement and evaluation systems.

To analyse the customer data, they were divided into ten annual cascades, except for people between 18-20 and people over 80. Customers under the age of 18 are not eligible to order. This results in eight groups. By defining young from 18 to 50 years, four associated groups are obtained. The definition old also results in four groups, which facilitates a comparison based on the same number of groups. In the methodology, the data were evaluated according to the number of orders. Changes in the survey parameters, for example turnover, can lead to different results. The analysis according to order numbers offers the advantage that in a turnover analysis, high-priced medicines can distort the results.

Following the quantitative analysis with empirical data, ten qualitative expert interviews were conducted to discuss the results of the quantitative analysis and add causalities to them. The expert interviews were conducted with Ms. Inna Zarayska (administrator) and other employees of the mail-order pharmacy under investigation. 
Due to their positions, access to data and experience, the experts can contribute further information and assessments as well as clarify open questions and discuss results.

An extensive literature search was conducted to ascertain the current state of research, legal rulings, and other results. The annual reports of the Federal Association of German Pharmacists (ABDA) serve as important literature; these contain key figures for all German pharmacies (approx. 19,000 pharmacies, as of 2019). Equally important key figures are provided by the Union and Constitutional Analysis by Dettling (Dettling, 2018). Furthermore, magazines such as the Pharmazeutische Zeitung and the Deutsche Apotheker Zeitung were consulted. These are designed for the pharmacy's sphere of activity in terms of the reproduction of expert opinions through quotations and interviews, current court rulings and legislative amendments, as well as published key figures. Furthermore, Google Search and Google Scholar Search were used. For data protection reasons, the expert interviews were anonymised (except for Ms Zarayska) and the mail-order pharmacy under investigation was given the fictitious name MedicineGo.

\section{Results}

For the creation of an economic customer profile of online customers for medicines, a sales channel analysis was evaluated. Customers of the MedicineGo mail-order pharmacy have the possibility to place their orders on different (six) sales channels:

- Webshop direct (homepage)

- Webshop indirectly (through search engine use)

- Phone

- E-mail

- Fax

- Letter

Using a quantitative method, an overview of customer orders by sales channel was created. The analysis of the MedicineGo raw data about the ordering behaviour of medicines in e-commerce showed that about $72 \%$ of the customer's order directly via the web shop, thus this represents the most important sales channel. 6\% access MedicineGo via various search engines and 21\% of customers order by phone (Fig. 2). The sales channels e-mail, fax, and letter account for less than 1\% of incoming orders, so they are economically negligible and only serve as a supplement.

The sales channels via the internet account for $78.38 \%$ (71.70\% direct webshop, $6.10 \%$ indirect webshop and $0.58 \%$ e-mail). The telephone sales channel is used by $21.42 \%$ of customers and is therefore the second most used sales channel and is also very important (see Fig. 2). The gender distribution included in the sales channel analysis does not show any significant differences between men and women regarding the sales channel

used. 


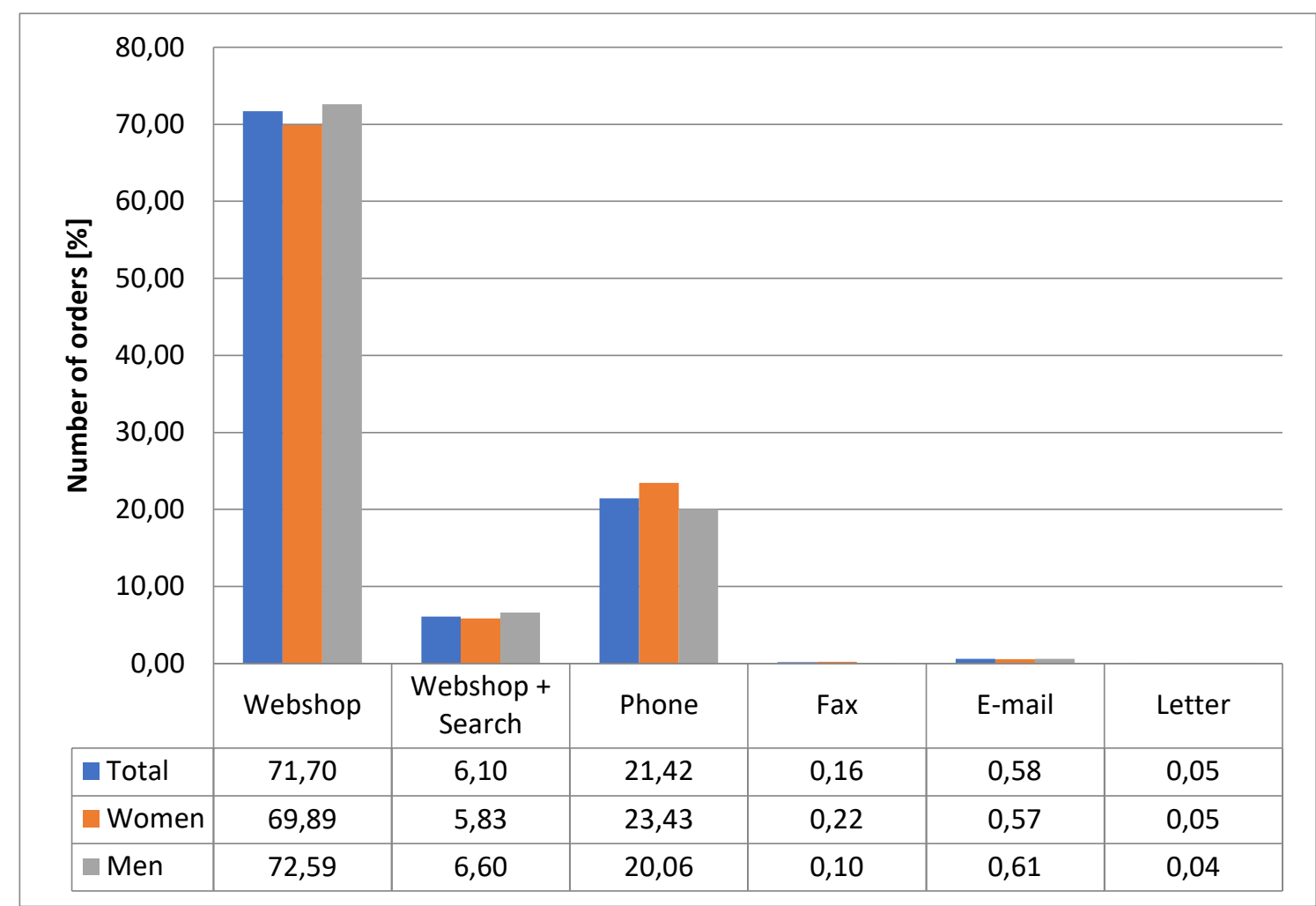

Fig. 2: Percentage of orders by sales channel with gender distribution included.

To determine the customer profile, a gender-specific analysis of the incoming orders was carried out. The analysis showed that about 53\% of the orders are from women, about $44 \%$ from men (Fig. 3). Just under 3\% of the orders cannot be assigned to a specific gender; this includes orders for company names, for example, which are gender neutral.

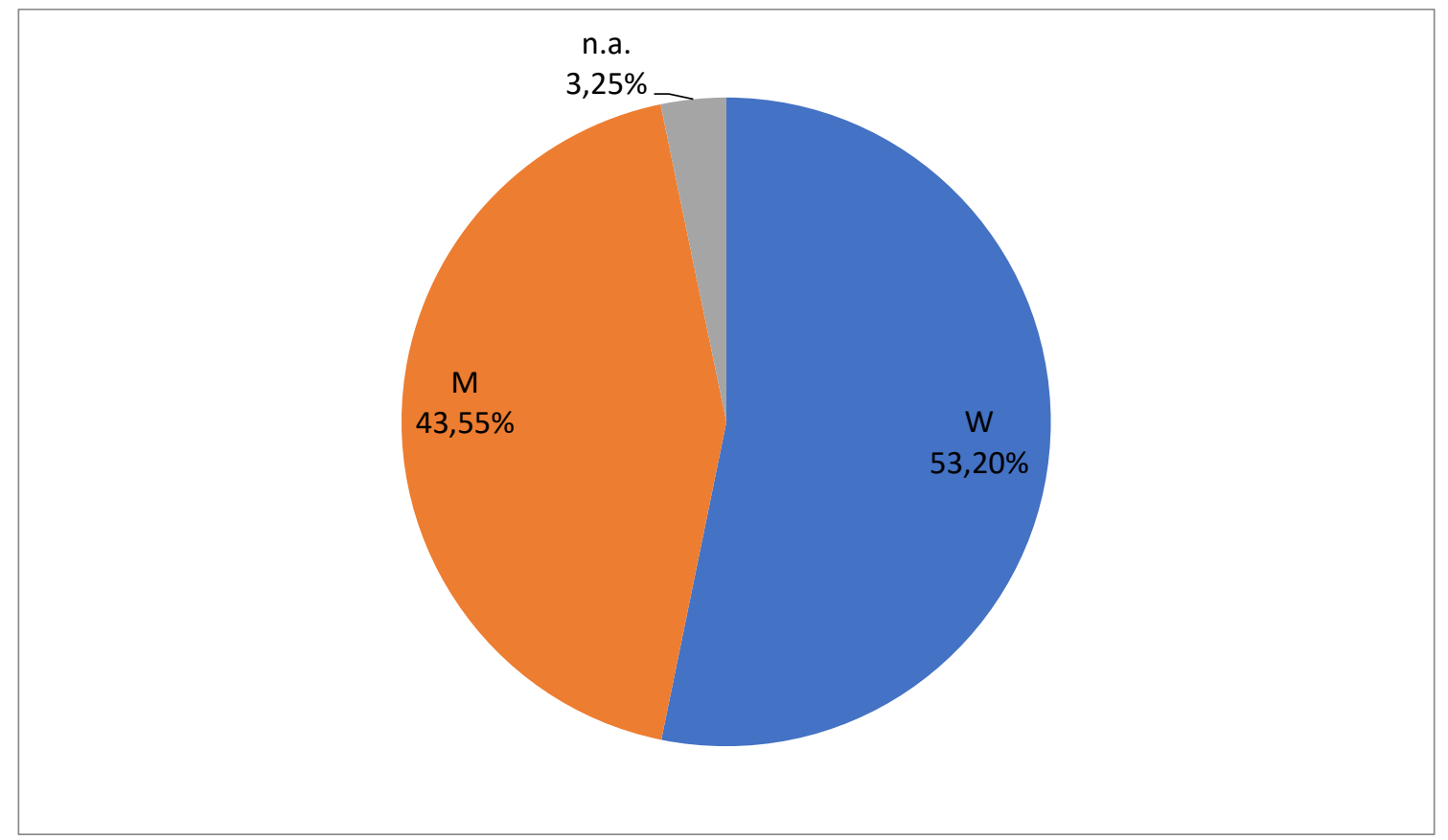

Fig. 3: Gender-specific share of orders across all sales channels. 
For a more detailed analysis of consumer behaviour and customer characteristics by means of quantitative methods, in addition to the sales channel analysis and the evaluation of the percentage gender distribution, customers were classified according to age (age classification in 10-year groups, exceptions are the age groups 18-20 and the over 80s). The overview of the percentage distribution of orders, considering an agespecific differentiation, serves to compare the age groups according to the number of orders and gives an indication of the order volume and the importance or weighting of individual age cascades. Regarding the customer structure analysis and the characterisation of online pharmacy customers, Fig. 4 represents the results:

- The group of 18- to 20-year-old customers: This is the smallest group, both in terms of the corresponding age figures / age cascade, and with about $0.06 \%$ of orders - a clear difference to the other age groups. According to the qualitative expert interviews with, orders in this age group are preferably placed on behalf of parents or grandparents. Accordingly, the share is very low.

- 21- to 30-year-old customers: Just under $1.4 \%$ of MedicineGo drug orders were placed by this age group in the defined period.

- From 31 to 40 years: The share of this age group is $6.1 \%$.

- 41 to 50 years: Here the group grows to $18.2 \%$.

- 51- to 60-year-old customers: At 24.1\%, this is the largest group; accordingly, most orders are placed by customers aged 51 to 60 , who belong to the old group.

- 61- to 70-year-old customers: This is the second largest group with an order share of $20.1 \%$.

- 71- to 80-year-old customers: MedicineGo mail-order pharmacy patients aged 71 to 80 are the third largest group with an order share of about $20.0 \%$.

- $\quad$ The age group of patients over 80 years is almost 6.1\%. This group is about the same size as the 31-40-year-old clients.

- For just under $4.1 \%$ of the customers, no age information was given. 


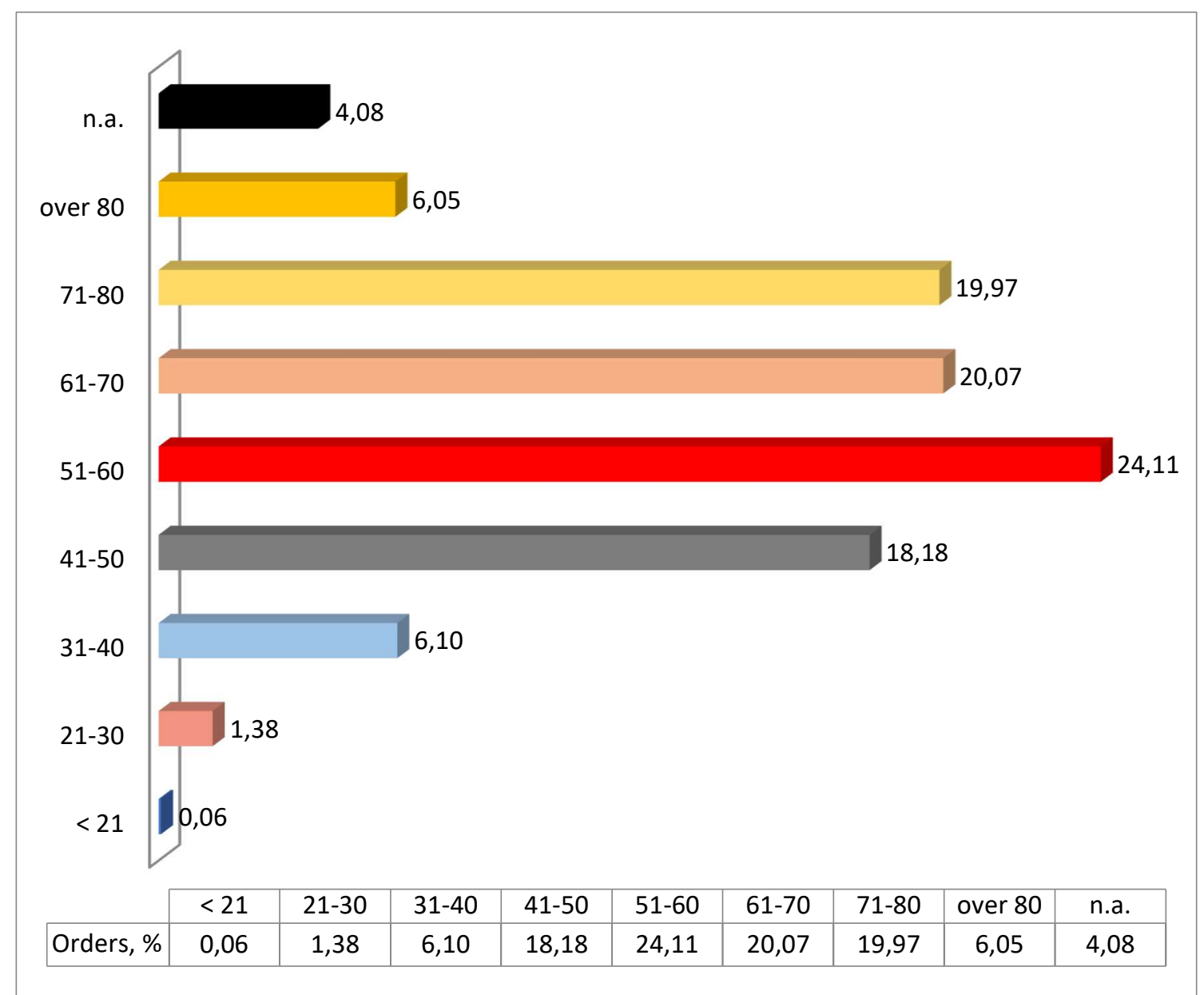

Fig. 4: Percentage distribution of orders taking into account age-specific differentiation.

The results of the quantitative analysis regarding the percentage distribution of order shares (Fig. 4) show that the three largest customer groups (by number of orders) are aged between 51 and 80. According to the established definition of young to old, the analysis yields a result of $1: 2.73$.

Regarding customer analysis in e-commerce for pharmaceutical orders, the use of sales channels was analysed depending on the age groups of customers. Fig. 5 shows the development of the three most important sales channels depending on the age of the customers - the following trends can be identified from this. As the age of the customers increases, the sales channel of direct web shop orders decreases. Regarding the sales channel, the two groups of 18-20-year-olds as well as 21-30-year-olds show somewhat lower values, so that the trend is recognisable, but these two groups form slight outliers. Another trend with increasing age is the decrease in indirect web shop orders - an exception is the customer group of 18-20-year-olds, this age category only orders directly via the web shop. A clear result is the increase in telephone orders with increasing age. The quantitative analysis shows that the telephone sales channel plays a subordinate role for customers aged 18-40. According to the expert interviews, telephone orders are increasingly perceived by older customers. They want to buy medicines at a low price and still do not want to do without a personal conversation and pharmaceutical advice. Furthermore, telephone ordering serves to find medicines more quickly.

The choice of sales channel used is independent of gender. However, the results show a strong affinity in the choice of sales channel depending on customer age. 


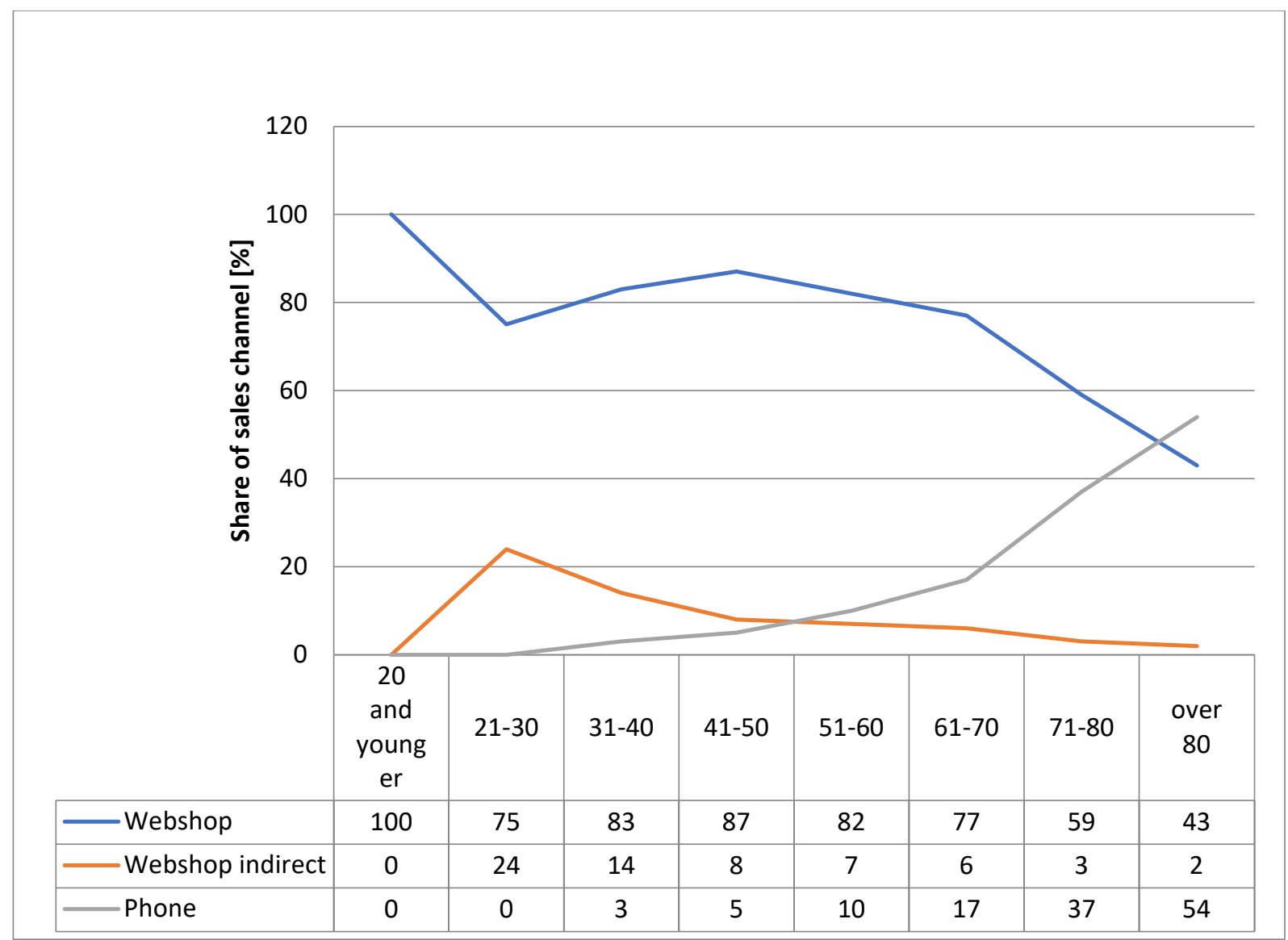

Fig. 5: Percentage of sales channel use depending on customer age.

Using the software SPSS, the correlation of the birth cohorts to the orders of the webshop sales channel was calculated directly 2-sided. The result of

-0.336 means an opposite correlation: the older the customers, the less the sales channel webshop is used directly. The correlation is at the 0.001 level and thus statistically significant (see Fig. 6).

\begin{tabular}{|c|c|c|c|}
\hline & & Year of birth & $\begin{array}{c}\text { Sales } \\
\text { channel } 1 \\
\text { (web shop } \\
\text { direct) }\end{array}$ \\
\hline \multirow[t]{3}{*}{ Year of birth } & $\begin{array}{l}\text { Pearson } \\
\text { correlation }\end{array}$ & 1 &,$- 336^{\star *}$ \\
\hline & Sig. (2-sided) & & 0,000 \\
\hline & $\mathrm{N}$ & 22595 & 22564 \\
\hline \multirow{3}{*}{$\begin{array}{l}\text { Sales } \\
\text { channel } 1 \\
\text { (web shop } \\
\text { direct) }\end{array}$} & $\begin{array}{l}\text { Pearson } \\
\text { correlation }\end{array}$ &,$- 336^{* *}$ & 1 \\
\hline & Sig. (2-sided) & 0,000 & \\
\hline & $\mathrm{N}$ & 22564 & 22564 \\
\hline
\end{tabular}

Fig. 6: Pearson correlation analysis (web shop/customer age).

Regarding the customer characterisation of pharmaceutical orders in e-commerce using the example of the German mail-order pharmacy MedicineGo, more women (53\%) order than men (44\%), 3\% are not specified. According to qualitative analysis, more women take over when ordering for their families, so that the proportion of women is higher. 
Most orders are received from customers aged 51-60 (order share 24.11\%). Overall, the three largest age groups are 51-60 years (24.11\%), 61-70 years $(20.07 \%)$ and $71-80$ years $(19.97 \%)$ and have a total share of incoming orders of $64.15 \%$. Based on empirical data and quantitative methods, the result is a ratio of 1:2.73 according to the proportion of orders in e-commerce from young to old - thus the hypothesis formulated in the objective is refuted.

\section{Discussion and summary}

The results of the quantitative analysis have shown that direct web shop orders $(72 \%$ by order share) and telephone orders (21\% by order share) are the two most important sales channels of a pharmaceutical mail order company. With almost $72 \%$ direct webshop orders, there is a clear gap to the second most common sales channel (telephone, 21\%). This is an important indicator that customers must be familiar with the web shop, due to the direct access. This indicates the importance of previously placed advertising measures. With the inclusion of content analyses of the expert interviews, it could be added that with increasing age, the telephone sales channel is gladly used, since a direct conversation facilitates the search for medicines and value is placed on a personal conversation. The customers who use search engines, i.e., do not go directly to MedicineGo's web shop, have an order share of approx. 6\%. These customers search for medicines online, for example via search engines with a price comparison function such as Medizinfuchs and can then be redirected to MedicineGo. It is difficult to evaluate the result of $6 \%$, as these customers may use the web shop directly when ordering again. There is no data available on whether a customer searches for medicines again via various search engines or orders directly via the already known web shop in case of a new need for medicines. It should also be added that the competitive situation in online trade is very great. The multitude of different offers is enormous due to the large variety of products. This could explain the low share of only $6 \%$ due to the large number of competitors.

Quantitatively, it was determined that the order share of women is higher $53 \%$ to $44 \%$ ). The reason for this could not be answered quantitatively, as no data was available. The qualitative analysis showed that more women order for their families, which explains the higher proportion. The higher proportion of women is interesting and could be considered to optimise advertising strategies. It remains to be mentioned that the number of orders was recorded - the influence of sales was not included. This requires further analysis.

To answer the initial hypothesis that when customers order medicines on the Internet, they are mainly between 18 and 50 years of age, based on the assumption that younger people (18 to 50 years) prefer to order online because they are more familiar with computers, an analysis of the percentage distribution of orders, taking age-specific differentiation into account, was used. The result showed a ratio of young (18-50 years) to old (over 50 years) of 1:2.73. In addition, the three largest order groups were identified, people aged 51 to 80 years - thus disproving the hypothesis. Customers aged 18 to 30 are the two smallest groups, so these offer great potential for growth.

According to quantitative analysis, the group of 21-30-year-old customers use the web shop most (indirectly), i.e., via search engine use. These usually have a price search function or are sorted by price. This is an indication that this age group (21-30 years) is the most price-conscious when ordering medicines.

To answer the research question, the customer profile of the national mail-order pharmacy MedicineGo shows a $72 \%$ use of the direct web shop as the most used sales 
channel, a higher proportion of women in terms of incoming orders of $53 \%$ and the three most important age groups are between 51 and 80 years old.

The fact that the demand for medicines also increases with age is not considered. In addition, the age groups were evaluated according to order share; the order volume according to turnover is not considered. Thus, conclusive statements on the importance of the individual age groups by turnover can only be made through further analyses. The data available on studies of online pharmacies in Germany, especially on ordering behaviour as well as the creation of customer structures, is limited. Depending on the attractiveness of the web shop and the level of awareness, the results of the individual mail-order pharmacies can vary. The results of the German mail-order pharmacy MedicineGo can only serve as an example. An exchange of several mail-order pharmacies by means of quantitative analysis could offer further insights.

\section{List of abbreviations}

Fig.

ABDA

Illustration

AMPreisV

Federal Association of German Pharmacist Associations

Pharmaceutical Price Ordinance

cvs

comma-separated values

E-mail

Electronic mail

e-prescription

electronic prescription

ECJ

European Court of Justice

$\mathrm{Rx}$

Prescription medicines

s.

see 


\section{References}

ABDA (2020). The pharmacy. Figures - Data - Facts 2020.

pharmacy adhoc (2020). SCHMIDT HOPES FOR VOASG EFFECT. 221 pharmacies less. Available at https://www.apotheke-adhoc.de/nachrichten/detail/politik/221apotheken-weniger-apothekenzahl-abda-schmidt/ [23.11.2020].

DETTLING, H.-U. (2018). Arzneimittelversorgung und flexibles Versandverbot für verschreibungspflichtige Arzneimittel. A Union and constitutional analysis. Eschborn: Govi.

ECJ, Judg. v. 19.10.2016, Deutsche Parkinson Vereinigung e.V., C-148/15.

KASKE (2019): E-prescription study 2019. Available at https://drkaske.de/studien/erezept-studie-2019/ [07.09.2019].

TEBROKE, E. (2017). OTC mail order: Shop pharmacy with sales boom. Pharmazeutische Zeitung, 5, 8.

ZTG (2020). E-prescription coming in July 2021. Verfügbar unter https://www.ztgnrw.de/2020/07/im-juli-2021-kommt-das-e-rezept/ [11.09.2020]. 